\title{
Resistance of common spring wheat genotypes to abiotic and biotic stresses in the Southern Urals
}

Kushnirenko I.Yu.*, Shreyder E.R., Bondarenko N.P.

Chelyabinsk Scientific Research Institute of Agriculture, Timiryazevsky, Russia

*email: ikush2001@mail.ru

Creation of genotypes with complex resistance to extreme environmental conditions, primarily to hydrothermal stresses and fungi diseases, is the main directions of the breeding program in Chelyabinsk Scientific Research Institute of Agriculture. The combination of alternative traits such as tolerance to drought and resistance to excessive moisture exceeding optimal norms is a one of methodological task. Long-term studies have shown that, while maintaining sensitivity to drought in a variety, nonspecific resistance to carbohydrate-protein depletion of seeds, lodging, and damage by rust species is induced. Long-term studies have shown that nonspecific resistance to carbohydrate-protein depletion of seeds, lodging and damage by rust species is induced in varieties sensitive to drought.

The cultivar Erythrospermum 59, that has a unique combination of drought tolerance with resistance to excessive moisture is the sample of optimal ecotype of spring wheat for conditions of Southern Urals. Its use as a source of high plasticity provides promising breeding material and more modern commercial varieties Duet, Chelyaba stepnaya, Chelyaba jubilee.

The use of the intraspecific genetic potential of common wheat does not provide a sufficient level of protection from leaf rust. In this case the new sources of resistance carrying foreign gene translocations from Secale sereale, Aegilops speltoides, Agropyron elongatum, Agropyron intermedium, Aegilops tauschii, Triticum ventricosum are involved in breeding programs. As a result, a series of varieties with leaf rust gene LrSp from Aegilops speltoides were created (Chelyaba 75, Chelyaba 80, Odintsovskaya, Ilmenskaya) was introduced. Using effective combination of Lr-genes that have lost their efficiency in the region, the variety Silach was created. Its high resistance to the South Ural population of Puccinia triticina is controlled by a combination of genes Lr10, Lr19 and Lr26. Monitoring of the racial composition and virulence of the regional population of leaf rust pathogen is carried out for successful breeding.

Along with these varieties, a promising selection of spring wheat has been created, combining resistance to abiotic stresses with resistance to leaf and stem rust and blotches. Using molecular markers, it was shown that the resistance genes in these lines differ from the known effective ones, transferred from alien species. The most promising lines are planned to be transferred to the State Variety Testing. The influence of promising spring wheat lines bearing alien translocations on the variability of pathogen populations by virulence was not observed, which will contribute to the stability of the genetic protection in the conditions of the Southern Urals.

Acknowledgements: The current research was supported by RFBR grant No. 19-01600052. 